\title{
rupture d'un rideau de palplanches
}

\author{
par \\ H. Josseaume \\ Laboratoire Central des Ponts et Chaussées \\ J. Jardin \\ Laboratoire Régional de l'Ouest Parisien
}

\section{Introduction}

Une importante voie routière de la région parisienne longe la rive droite d'une rivière sur un remblai étayé latéralement par un rideau de palplanches jouant le rôle de soutènement de rive. Dix-huit mois après la mise en service de cette voie routière, le flanc du remblai surplombant la rivière s'effondrait sur une longueur d'environ $80 \mathrm{~m}$, tandis que le rideau de palplanches subissait des déplacements de plusieurs mètres d'amplitude qui provoquaient sa destruction.

Le Service de l'Équipement, Maître d'CEuvre, a confié au Laboratoire Régional de l'Ouest Parisien et au Laboratoire Central des Ponts et Chaussées la mission de déterminer les causes de cette rupture et de proposer des méthodes permettant de réparer l'ouvrage dans sa partie endommagée et éventuellement de le conforter dans sa partie encore intacte. On rend compte ci-après des études effectuées dans le cadre de cette mission.

\section{Description du site avant la rupture}

Dans la zone intéressée par la rupture, le remblai routier s'appuie sur un sol dont la stratigraphie est classique en région parisienne (fig. 1):

- en surface, des remblais d'apport constitués de matériaux de décharge et de démolition (limons, argiles, blocs) recouvrant des alluvions récentes fines (limons argileux plus ou moins organiques);

- des alluvions anciennes, formées pour l'essentiel de sables grossiers relativement argileux relayés à peu de distance du lit de la rivière par une couche d'éboulis (sables, marne et blocs);

- les sables de Beauchamp, dont le faciès argileux est largement prédominant, le faciès gréseux n'apparaissant à la partie supérieure de la couche qu'à une certaine distance de la rivière;

- les marnes et caillasses.
Le remblai routier, construit en matériau sableux et en grave-ciment, est limité latéralement côté rivière par un talus de pente $\frac{1}{1,4}$ et de hauteur $5,50 \mathrm{~m}$ dont le pied prend appui sur un soutènement de rive en palplanches. Celui-ci était initialement constitué de palplanches $S \ell_{2}$ de longueur $4,10 \mathrm{~m}$. II était ancré en tête par une ligne de tirants d'ancrage à plaque pivotante espacés de 4,5 m. Ces tirants, qui avaient une longueur d'environ $10 \mathrm{~m}$ étaient inclinés à $30^{\circ}$ sur l'horizontale et prenaient appui dans le sable de Beauchamp.

\section{Observations après l'accident}

L'accident, qui s'est produit alors que le niveau de la rivière se situait à $0,8 \mathrm{~m}$ au-dessous de la tête du rideau a eu pour conséquences :

- un déplacement horizontal important dont l'amplitude a atteint $6 \mathrm{~m}$ dans la partie centrale de la zone endommagée. Ce déplacement s'est traduit par un vrillage du rideau, dont la tête s'est inclinée tantôt vers la rivière, tantôt vers la rive;

- un glissement du remblai sur une longueur de $80 \mathrm{~m}$, qui a provoqué l'effondrement de sa plate-forme jusqu'à environ $7 \mathrm{~m}$ de la crête du talus et l'apparition de fissures parallèles à celle-ci en arrière de la zone effondrée.

Par ailleurs, un important suintement apparaissant dans le remblai, sensiblement au milieu de la zone effondrée, a mis en évidence une fuite dans une canalisation de distribution d'eau potable située à $2,50 \mathrm{~m}$ sous la chaussée et longeant la rivière à environ $8 \mathrm{~m}$ en arrière de la crête initiale du talus. Selon la Compagnie Générale des Eaux, cette fuite était antérieure d'au moins 6 mois à la rupture du remblai, et son débit pouvait être estimé à $1 \mathrm{l} / \mathrm{s}$.

En outre, au cours des investigations qui ont été faites aux abords de la zone accidentée, on a constaté en amont de celle-ci, des fissures dans la chaussée 

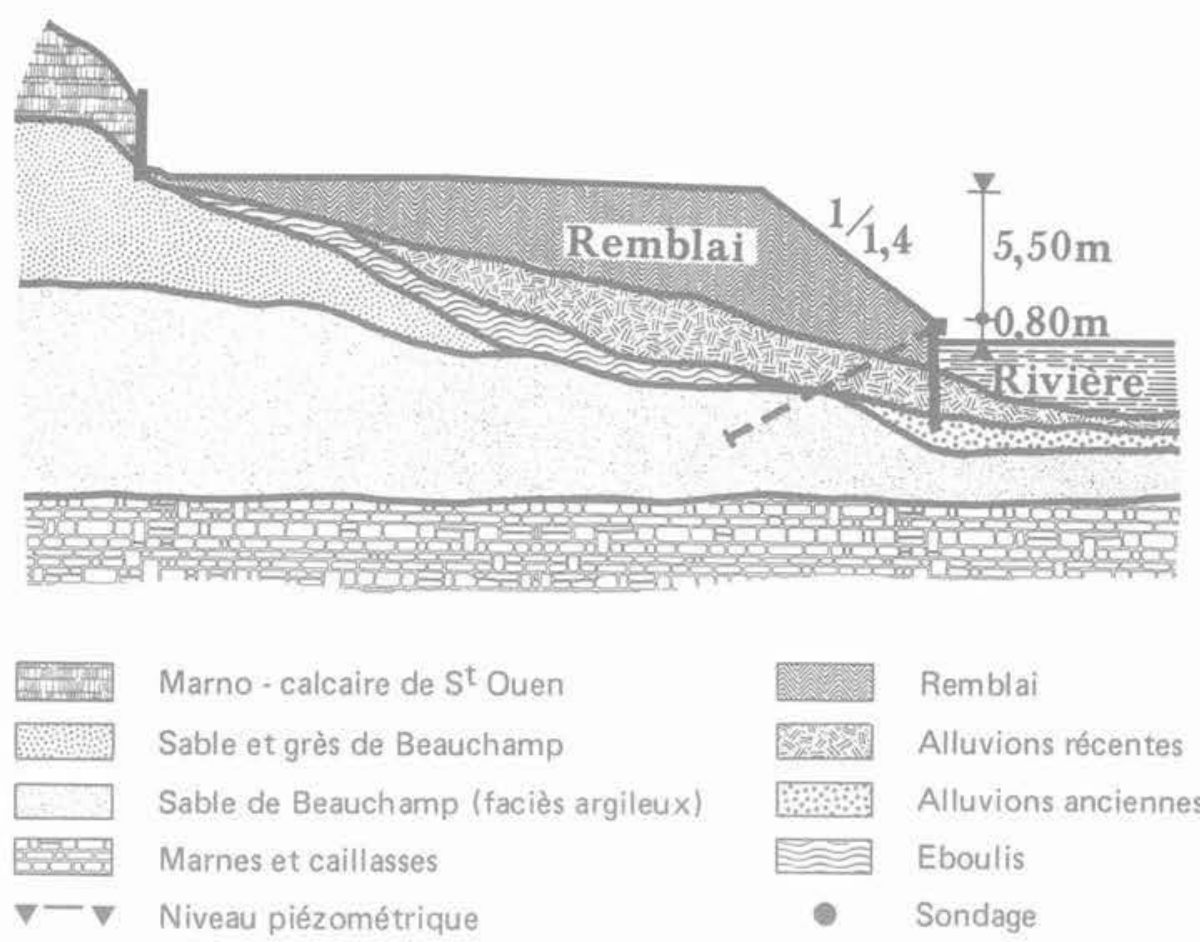

Marno - calcaire de $S^{t}$ Ouen

Sable et grès de Beauchamp

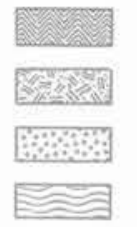

Remblai

Sable de Beauchamp (faciès argileux)

Alluvions récentes

Marnes et caillasses

Alluvions anciennes

Eboulis

$\nabla-\nabla \quad$ Niveau piézométrique

- Sondage

Fig. 1 Coupe de la berge de la rivière et du remblai routier dans la zone de rupture

orientées parallèlement à la rivière (fig. 2). Apparaissant à environ $80 \mathrm{~m}$ de la limite du glissement, elles s'étendaient jusqu'à $200 \mathrm{~m}$ de celui-ci et tendaient alors à s'orienter perpendiculairement à la rivière, laissant craindre une nouvelle rupture. Une surveillance systématique des déplacements de repères topographiques implantés dans la zone des fissures, sur la chaussée et sur la poutre de couronnement du rideau, a montré que cette dernière ne subissait pas de déplacement appréciable mais a mis en évidence des tassements de la plate-forme du remblai.

\section{4 Étude géotechnique}

L'effondrement du remblai pouvait être imputé soit à un défaut de stabilité du rideau de palplanches ayant eu pour effet d'amorcer la rupture, soit à un glissement de la rive qui aurait entraîné le rideau dans son mouvement. L'éventualité du développement d'un fontis dans les marnes et caillasses, sous le talus du remblai, ne pouvait en outre être exclue a priori.

La recherche des causes de l'accident impliquait alors la connaissance des éléments permettant l'analyse de la stabilité du rideau et de celle de la rive, c'est-à-dire :

- la coupe du sol dans la zone effondrée et à son voisinage immédiat;

- les conditions hydrauliques;

- les caractéristiques mécaniques du sol et plus particulièrement ses paramètres de résistance au cisaillement.

Des sondages généralement équipés en piézomètres, ont donc été réalisés dans trois profils perpendiculaires à la rivière. Le profil 1 (7 sondages) était implanté dans la partie centrale de la zone effondrée, les profils 2 ( 5 sondages) et 3 ( 4 sondages) en amont de cette zone, l'un à $40 \mathrm{~m}$ et l'autre à $150 \mathrm{~m}$ de son extrémité (fig. 2). Le dernier était situé dans la section où l'apparition de fissures faisait redouter une seconde rupture. En règle générale, ces sondages ont été arrêtés à la partie supérieure des marnes et caillasses. Cependant, au profil 1 trois sondages ont entièrement traversé cette formation afin d'y déceler un éventuel fontis.

Des essais de perméabilité Lefranc à charge variable ont été effectués au niveau du remblai, des alluvions et du sable de Beauchamp lors de l'exécution des sondages dans les profils 2 et 3 . Des échantillons intacts ont été prélevés dans ces formations afin de déterminer leurs caractéristiques d'identification et leur résistance au cisaillement.

\subsection{Stratigraphie}

L'exploitation des sondages a permis de définir avec précision la géométrie des couches au droit de chaque profil. L'examen des coupes correspondantes fait apparaître (fig. 3) :

- que la rupture n'a affecté que les couches superficielles, remblais et alluvions, qui ont été entièrement désorganisées (fig. 3 a). Ceci, joint au fait que les sondages profonds n'ont indiqué aucune anomalie dans les marnes et caillasses, a conduit à écarter l'hypothèse selon laquelle un fontis aurait été à l'origine de l'accident;

- une bonne homogénéité du site dans la zone étudiée, principalement au niveau des couches superficielles, les coupes relatives aux profils 2 et 3 (fig. $3 b$ et $3 c$ ) étant très semblables. 


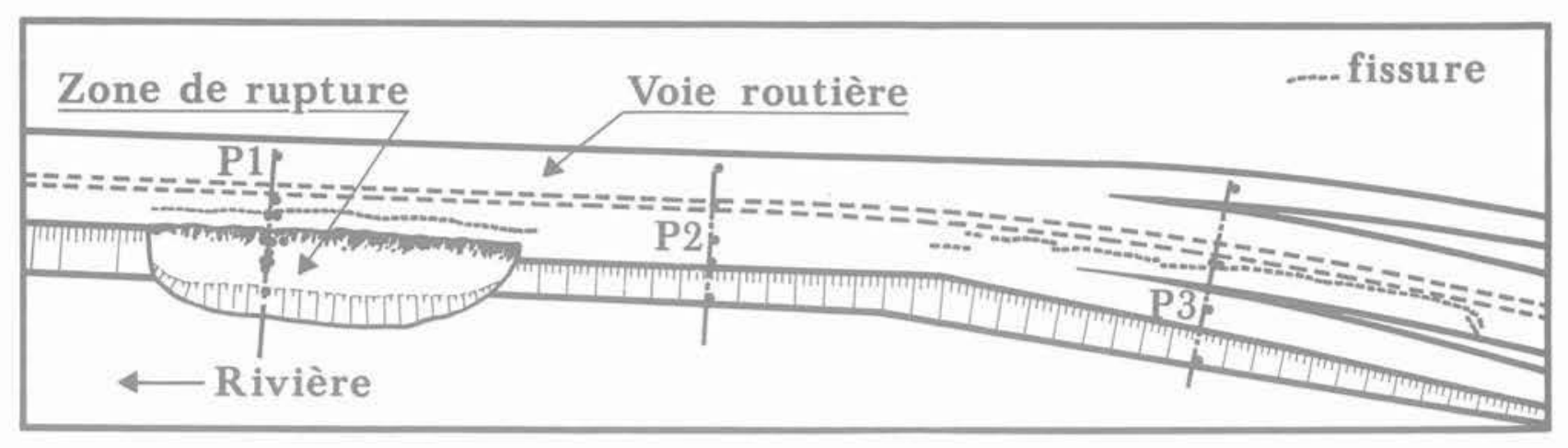

Fig. 2 Vue en plan de la zone de rupture et implantation des profils de sondages
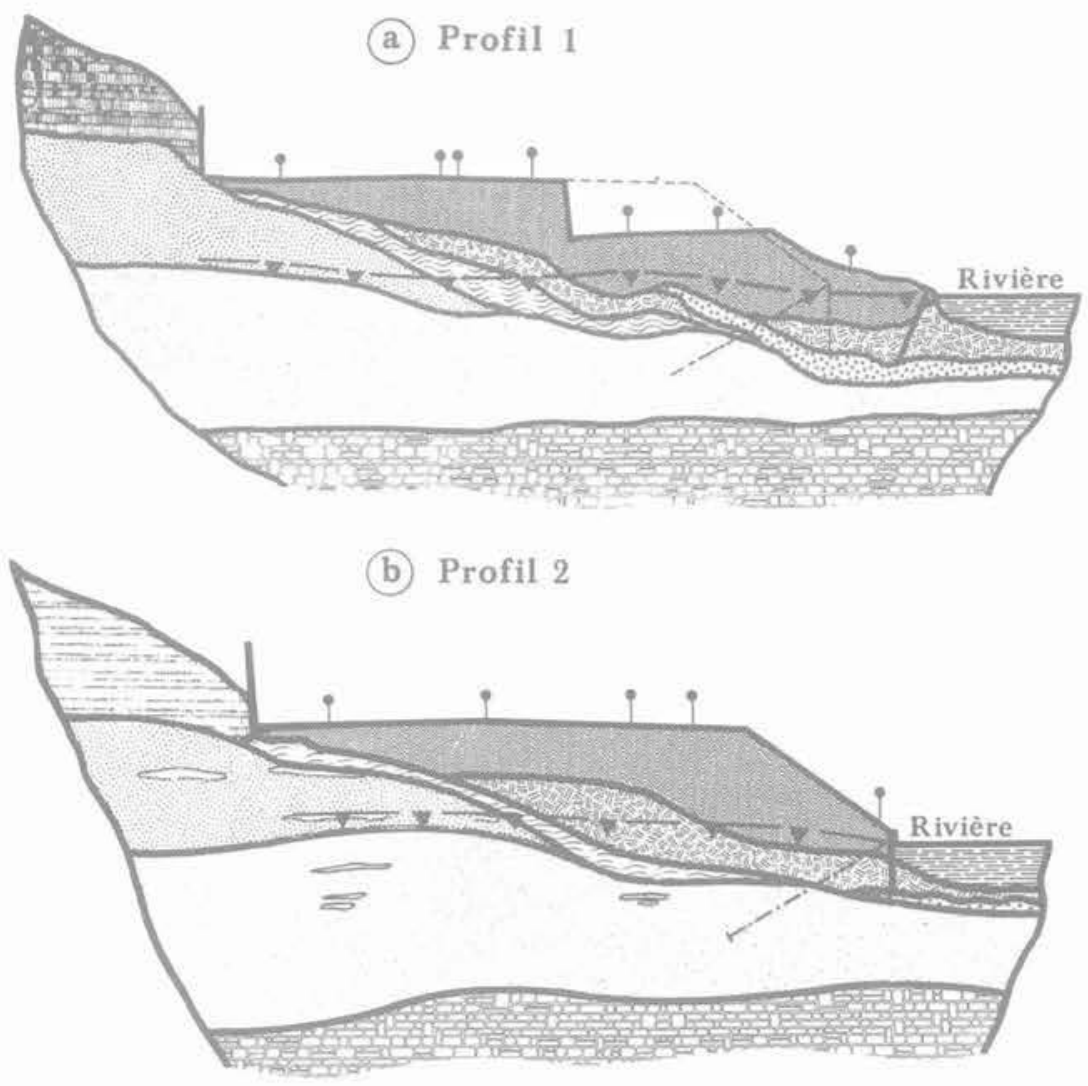

(c) Profil 3

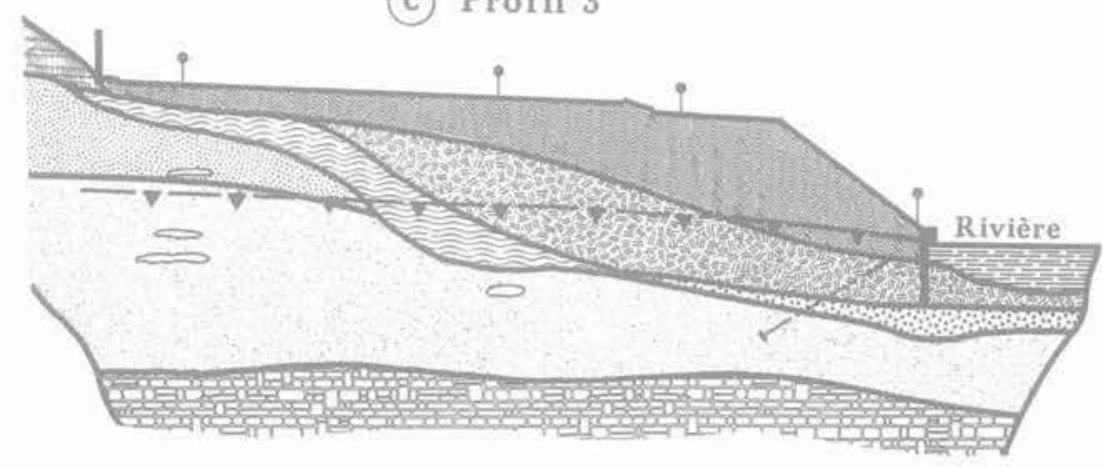

\begin{tabular}{|c|c|c|c|}
\hline 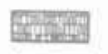 & Marno - calcaire de $S^{t}$ Ouen & & Remblai \\
\hline & Sable et grès de Beauchamp & 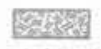 & Alluvions récentes \\
\hline & Sable de Beauchamp (faciès argileux) & 丽如 & Alluvions anciennes \\
\hline & Marnes et caillasses & $\approx$ & Eboulis \\
\hline & Niveau piézométrique & $\bullet$ & Sondage \\
\hline
\end{tabular}

Fig. 3 Coupes du sol au droit des trois profils de sondage 


\subsection{Caractéristiques géotechniques}

Les caractéristiques moyennes d'identification des alluvions fines et du sable de Beauchamp sont regroupées dans le tableau 1.

\begin{tabular}{c|c|c|c|c|c}
\hline Formation & $\begin{array}{c}\text { Poids } \\
\text { volumique } \\
\gamma\left(\mathrm{kN} / \mathrm{m}^{3}\right)\end{array}$ & $\begin{array}{c}\text { Teneur en } \\
\text { eau } \\
\mathrm{w}(\%)\end{array}$ & $\begin{array}{c}\text { Limite de } \\
\text { liquidité } \\
\mathrm{w}_{\mathrm{d}}(\%)\end{array}$ & $\begin{array}{c}\text { Indice de } \\
\text { plasticité } \\
\mathrm{I}_{p}(\%)\end{array}$ & $\begin{array}{c}\text { Teneur en } \\
\text { matières } \\
\text { organiques } \\
(\%)\end{array}$ \\
\hline $\begin{array}{c}\text { Partie } \\
\text { supérieure } \\
\text { des } \\
\text { alluvions } \\
\text { fines }\end{array}$ & 17.5 & 50 & 74 & 33 & \\
\hline $\begin{array}{c}\text { Partie } \\
\text { inférieure } \\
\text { des } \\
\text { alluvions } \\
\text { fines }\end{array}$ & 14 & 100 & 80 & 32 & 12 \\
\hline $\begin{array}{c}\text { Sable } \\
\text { de }\end{array}$ & 20 & 20 & 38 & 14 & \\
$\begin{array}{c}\text { Beauchamp } \\
\text { faciès } \\
\text { argileux }\end{array}$ & 20 & & & & \\
\hline
\end{tabular}

Tableau 1 Caractéristiques moyennes d'identification des alluvions fines et du sable de Beauchamp

La résistance au cisaillement drainé du sol a été déterminée à partir d'essais triaxiaux de type consolidé non drainé avec mesure de la pression interstitielle, effectués sur les échantillons qui ont pu être prélevés. Les essais, réalisés après saturation du sol sous une contre-pression de $200 \mathrm{kPa}$, ont donné les résultats suivants.

\subsubsection{Remblais : $\mathrm{c}^{\prime}=15 \mathrm{kPa}, \varphi^{\prime}=32^{\circ}$}

Ces valeurs, obtenues à partir de 7 éprouvettes de sol fin (les seules qui aient pu être taillées), ne peuvent être $\varphi^{\prime}=35^{\circ}$, comme représentatives de l'ensemble du matériau de remblai, très hétérogène. Le comportement de ce matériau se situe vraisemblablement entre celui d'un sol pulvérulent d'angle de frottement $\varphi^{\prime}=36^{\circ}$ (angle de talus du remblai) et celui d'un sol ayant un angle de frottement du même ordre ( 30 à $\left.36^{\circ}\right)$ avec en outre une cohésion élevée (supérieure à $15 \mathrm{kPa}$ ), le remblai n'étant généralement pas saturé.

\subsubsection{Alluvions récentes}

Les résultats sont extrêmement dispersés (fig. 4). Pour 14 des 21 éprouvettes essayées, les points représentatifs de l'état des contraintes effectives à la rupture se situent dans la plage délimitée par des droites intrinsèques telles que $c^{\prime}=0, \varphi^{\prime}=35^{\circ}$, d'une part et $c^{\prime}=15 \mathrm{kPa}, \varphi^{\prime}=40^{\circ}$, d'autre part. Les points relatifs aux 7 autres se distribuent à peu près également de part et d'autre de cette plage, la résistance minimale ne correspondant jamais à un angle de frottement interne inférieur à $29^{\circ}$.
II apparaît également que les résistances les plus élevées sont systématiquement obtenues pour les éprouvettes prélevées à la partie inférieure de la couche, peu dense et à forte tendance organique.

Zompte tenu de ces données et aussi du fait que .'h. stoire du sol (normalement consolidé ou légèrement surconsolidé) ne permet pas d'expliquer la cohésion indiquée par les essais, on a pu attribuer aux alluvions modernes les valeurs suivantes:

- $c^{\prime}=0, \varphi^{\prime}=40^{\circ}$ pour la partie inférieure de la couche $\left.l=14 \mathrm{kN} / \mathrm{m}^{3}\right)$;

- $\quad=0, \varphi^{\prime}=35^{\circ}$ pour la partie supérieure de la wouche $\left(\gamma=17,5 \mathrm{kN} / \mathrm{m}^{3}\right)$.

\subsubsection{Sable de Beauchamp : $c^{\prime}=0, \varphi^{\prime}=39^{\circ}$}

\subsection{Conditions hydrauliques}

Les reler śs piézométriques effectués dans les 3 profils ont n.o. ré que la surface piézométrique de la nappe des formations superficielles se confond avec celle de la nuppe du sable de Beauchamp qui l'alimente. Ces nappes sont drainées par la rivière et au cours des trois mois ayant suivi l'accident, leur surface au voisinage du rideau s'est maintenue sensiblement au niveau de la tête de ce dernier, du moins dans la zone non perturbée par la rupture.

Compte-tenu de l'homogénéité du site, les conditions de nappe devaient être sensiblement identiques dans la zone effondrée avant que ne se produise la rupture, à moins que la fuite survenue à la canalisation de distribution d'eau n'ait provoqué une remontée locale de la nappe.

Les essais de perméabilité en place ont montré que le coefficient de perméabilité des sols superficiels (alluvions récentes et anciennes, remblais) varie de $10^{-4} \mathrm{~m} / \mathrm{s}$ à $10^{-5} \mathrm{~m} / \mathrm{s}$. Le sable de Beauchamp, qui se caractérise par une perméabilité 100 à 1000 fois plus faible, peut être considéré comme imperméable vis-à-vis de ces formations.

\section{5 Études de stabilité}

\subsection{Caractéristiques géométriques et hydrauli- ques}

La coupe du sol prise en compte pour les études de stabilité est représentée sur la figure 5. La désorganisation des couches superficielles dans la zone de rupture n'ayant pas permis de reconstituer leur géométrie initiale à partir des seuls sondages implantés dans le profil 1, cette coupe a été établie à partir des données recueillies dans les profils 2 et 3 .

Le choix des hypothèses concernant la nappe à l'arrière du rideau était particulièrement important. Aucun dragage ou remblaiement susceptible de perturber l'équilibre du remblai n'ayant été entrepris avant l'accident et la surface de la rivière se trouvant au-dessus de son niveau moyen, la rupture ne pouvait guère trouver son origine que dans une modification des conditions hydrauliques à l'arrière du rideau.

Comme indiqué précédemment, la surface de la nappe arrière au voisinage du rideau devait se situer sensiblement à la hauteur de la tête des palplanches lors de l'accident, à moins que la fuite de la canalisation n'ait provoqué une remontée locale de la nappe. 


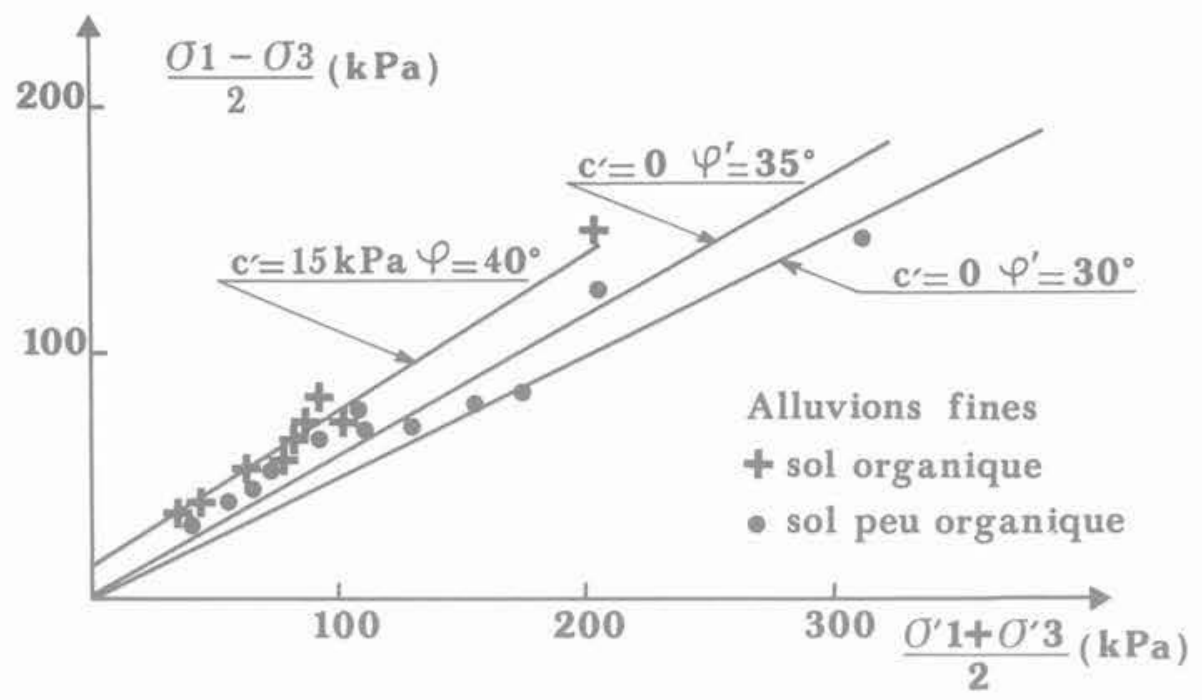

Fig. 4 Diagramme des contraintes effectives à la rupture obtenues dans les essais triaxiaux consolidés non drainés avec mesure de la pression interstitielle effectués sur les alluvions fines

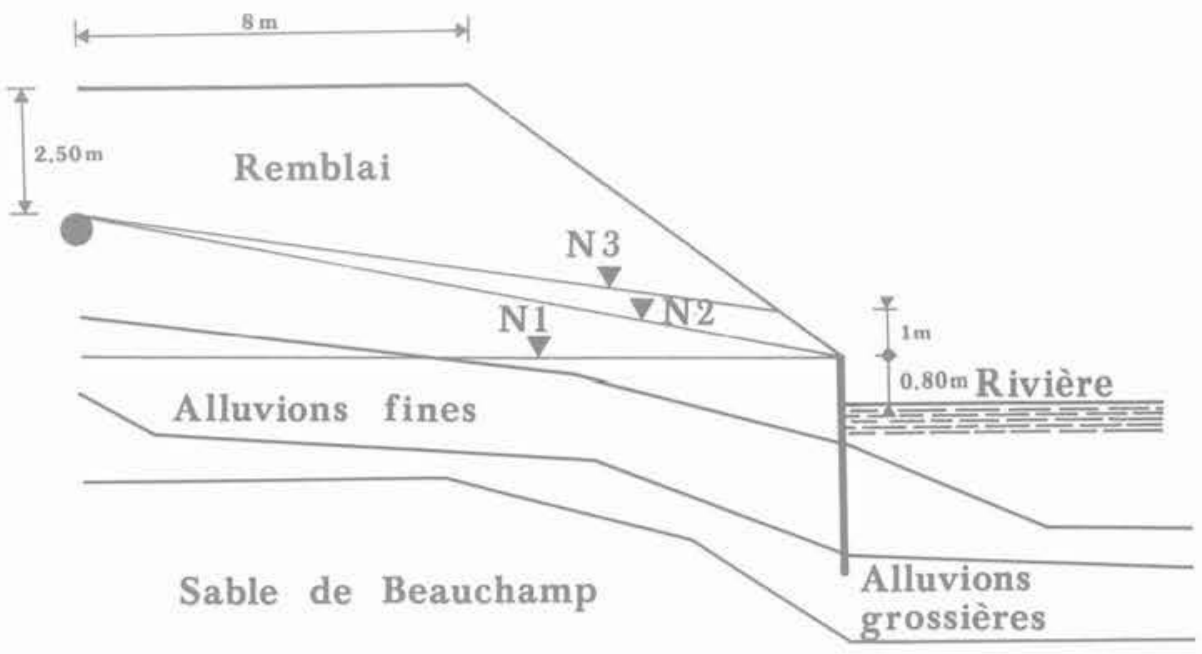

Fig. 5 Coupe du sol prise en compte dans les études de stabilité

Le débit de cette fuite étant connu approximativement, de même que les caractéristiques hydrauliques du site, il était théoriquement possible de déterminer par le calcul un ordre de grandeur de cette remontée. Mais le fait que l'écoulement créé par la fuite soit un écoulement tridimensionnel complexe interdisait pratiquement cette procédure. Aussi, pour étudier l'éventualité d'une remontée locale d'amplitude sensible de la nappe, a-t-on schématisé considérablement le problème. On a admis un relèvement uniforme de la nappe le long de la canalisation et l'on a comparé au débit total de la fuite le débit unitaire de l'écoulement plan qui s'établit alors dans un profil transversal. Dans I'hypothèse d'un relèvement de la surface de la nappe jusqu'au niveau de la canalisation au droit de cette dernière, le débit unitaire maximum déterminé par analogie électrique en attribuant aux alluvions et au remblai la plus forte des perméabilités mesurées en place, était très sensiblement inférieur au débit de fuite (au moins une quinzaine de fois). Il apparaissait donc, en dépit du caractère simpliste des hypothèses de l'étude, qu'une remontée locale de la nappe, d'amplitude ou d'extension importante, était très vraisemblable et même probable.

Cette éventualité a été prise en compte dans les calculs de stabilité qui ont été effectués en affectant successivement à la surface de la nappe arrière les positions $N_{1}$ (influence négligeable de la fuite), $N_{2}$ et $N_{3}$ (rôle important de la fuite) représentées sur la figure 5 . Les pressions interstitielles à l'arrière du rideau ont été calculées en assimilant les équipotentielles de l'écoulement à des verticales. 


\subsection{Stabilité de la rive}

L'accident pouvait être dû à l'instabilité propre de la berge de la rivière qui aurait alors glissé le long d'une surface passant sous le rideau et englobant le terrain sollicité par celui-ci. Cette éventualité était suggérée par l'extension de la zone effondrée en arrière du rideau. Elle a été étudiée en tenant compte du niveau de la rivière constaté lors de la rupture et en affectant successivement les positions $\mathrm{N}_{1}$ et $\mathrm{N}_{3}$ à la surface de la nappe arrière. L'étude de stabilité de la rive ayant été effectuée alors que l'on ne disposait que d'un petit nombre de résultats d'essais triaxiaux, des caractéristiques mécaniques pessimistes ont été attribuées aux différentes couches du sol:

$$
\begin{array}{ll}
\text { remblai } & \gamma=20 \mathrm{kN} / \mathrm{m}^{3}, \quad c^{\prime}=0, \varphi^{\prime}=32^{\circ}, \\
\text { alluvions anciennes } & \gamma=17,5 \mathrm{kN} / \mathrm{m}^{3}, \mathrm{c}^{\prime}=0, \varphi^{\prime}=30^{\circ}, \\
\text { alluvions modernes } & \gamma=17,5 \mathrm{kN} / \mathrm{m}^{3}, \mathrm{c}^{\prime}=0, \\
30^{\circ}=<\varphi^{\prime}<35^{\circ} .
\end{array}
$$

Les calculs ont été effectués au moyen du programme Vestal, qui permet l'étude de la stabilité des pentes en rupture non circulaire par la méthode des perturbations (Faure et al, 1975). Les surfaces de giissement envisagées se situaient au-dessus du toit du sable de Beauchamp. Elles n'englobaient donc pas l'ancrage des tirants dans cette formation et, de ce fait, ceux-ci exercaient une action stabilisatrice sur le volume de sol susceptible de glisser. Celle-ci n'a pas été prise en compte dans les calculs.

On a tout d'abord étudié la possibilité d'une rupture le long d'une surface de glissement correspondant sensiblement au contour de la zone effondrée, que les observations effectuées après la rupture permettaient de situer entre les lignes (A) et (B) représentées sur la figure 6. Les valeurs minimales du coefficient de sécurité obtenues le long de telles surfaces sont indiquées dans le tableau 2.

\begin{tabular}{c|c|c}
\hline $\begin{array}{l}\text { Alluvions } \\
\text { fines }\end{array}$ & Nappe & $\mathrm{N}_{1}$ \\
\hline$\varphi^{\prime}=30^{\circ}$ & $\mathrm{F}=1,44$ & $\mathrm{~F}=1,14$ \\
\hline$\varphi^{\prime}=35^{\circ}$ & $\mathrm{F}=1,64$ & $\mathrm{~F}=1,30$ \\
\hline
\end{tabular}

Tableau 2 Valeurs du coefficient de sécurité relatives aux surfaces de glissement situées dans le fuseau délimité par courbes (A) et (B)

En dépit du caractère pessimiste des données géotechniques et du fait que l'action stabilisatrice des tirants n'ait pas été prise en compte, elles étaient systématiquement supérieures à l'unité, ce qui indiquait clairement que l'accident ne trouvait pas son origine dans un glissement le long de surfaces telles que $(A)$ ou $(B)$.

Une recherche des surfaces de glissement les plus défavorables montrait qu'il fallait envisager des surfaces telles que (C) ou (D) recoupant la surface du sol à faible distance du rideau (fig. 6), pour que le coefficient de sécurité corresponde effectivement à l'éventualité d'une rupture. II s'ensuivait donc qu'un glissement de la rive n'avait pu se développer qu'au voisinage du rideau, c'est-à-dire dans une zone correspondant approximativement au prisme de Coulomb. L'origine de l'accident devait donc être recherché dans le comportement propre du rideau.

\subsection{Stabilité propre du rideau}

Si la rupture résultait d'une instabilité propre du rideau, cette dernière pouvait être liée soit à une insuffisance de la butée disponible sur la face avant du rideau, soit à une réaction d'ancrage trop élevée vis-à-vis de la capacité des tirants. L'analyse de la stabilité propre du rideau impliquait donc la détermination de la butée effectivement mobilisée sur la hauteur en fiche et de la réaction unitaire d'ancrage. L'une et l'autre ont été calculées en admettant que le rideau travaillait dans des conditions voisines de celles de la butée simple. Cette hypothèse était effectivement réalisée si la rupture était due à une fiche trop courte et elle n'introduisait qu'une erreur assez faible sur la réaction d'ancrage si le rideau travaillait en rideau semi-encastré.

Dans ces conditions, les efforts appliqués au rideau étaient (fig. 7) :

- la réaction d'ancrage, de composante horizontale $T$,

- la résultante des poussées du sol et de l'eau sur la face arrière, de composante horizontale $\mathrm{Pa}$,

- la poussée de l'eau Pw et la butée effective du sol, ayant pour composante horizontale $\mathrm{P}^{\prime} \mathrm{pm}$, sur la face avant.

Si l'on admet que le point d'application de la butée effectivement mobilisée est le même que celui de la butée à l'équilibre limite, l'équilibre du rideau fournit les expressions de $\mathrm{P}^{\prime} \mathrm{pm}$ et de $\mathrm{T}$;

$$
P^{\prime} p m=\frac{z_{a} P a-z_{w} P w}{z_{p}},
$$

$z_{a}, z_{p}$ et $z_{w}$ étant définis sur la figure 7 ,

$$
T=T_{1}=P a-P^{\prime} p m-P_{w}
$$

pour $P^{\prime} p m<P^{\prime} p \cos \delta p$,

$$
T=T_{2}=P a-P^{\prime} p \cos \delta p-P w
$$

pour $P^{\prime} p m \geqslant P^{\prime} p \cos \delta p$,

$P^{\prime} p$ étant la butée effective du sol sur la face avant du rideau, à l'équilibre limite et $\delta p$ son inclinaison sur l'horizontale.

$T_{\max }$ désignant la composante horizontale de la réaction limite d'ancrage, la stabilité du rideau impliquait :

$$
P^{\prime} p m<P^{\prime} p \cos \delta \text { et } T=T_{1}<T_{\max } \text {, }
$$

c'est-à-dire que les coefficients de sécurité vis-à-vis d'une insuffisance de fiche et vis-à-vis d'une insuffisance d'ancrage, respectivement égaux à :

$$
F p=\frac{P^{\prime} p \cos \delta p}{P^{\prime} p m} \text { et } F a=\frac{T_{\max }}{T},
$$

soient simultanément supérieurs à l'unité.

Le calcul de $\mathrm{Fp}$ et $\mathrm{Fa}$ a été fait en considérant le profil-type de la figure 5. Cependant, la profondeur de pénétration des palplanches dans les alluvions anciennes étant très faible, on a considéré que le rideau était entièrement fiché dans les alluvions fines. Les poussée et butée sur l'ouvrage ne dépendaient alors que des caractéristiques mécaniques du remblai et des alluvions fines. 


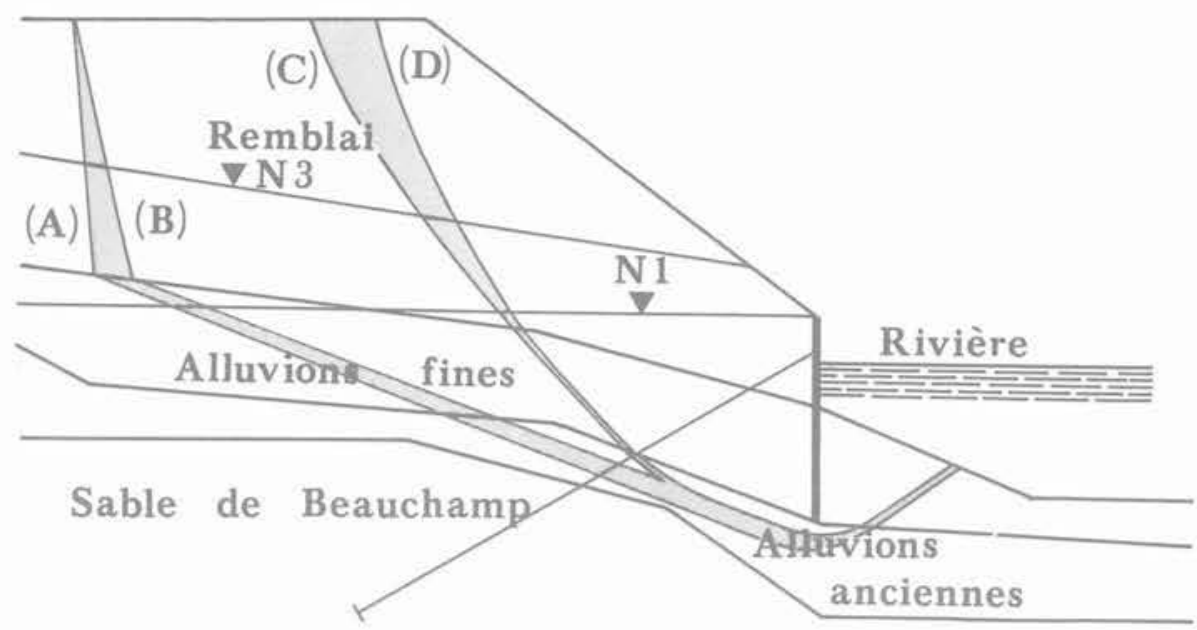

Fig. 6 Surfaces de glissement envisagées lors de l'etude de stabilité de la berge de la rivière

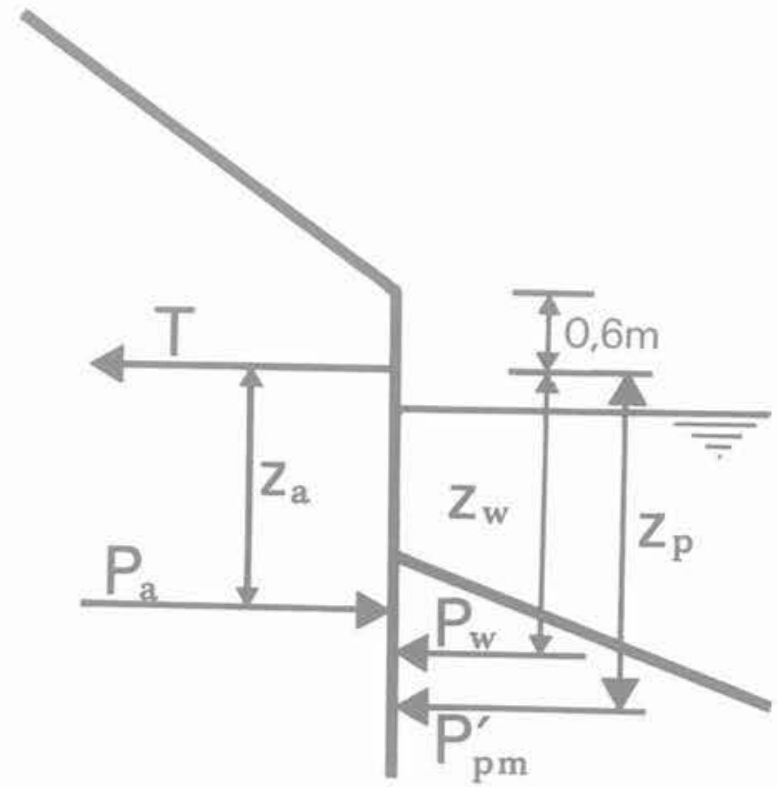

Fig. 7 Composantes horizontales des efforts appliqués au rideau

Le remblai a été considéré comme un sol sans cohésion d'angle de frottement interne $\varphi^{\prime}=36^{\circ}$, puis comme un matériau suffisamment cohérent pour n'exercer aucune poussée propre, c'est-à-dire jouant le rôle d'une surcharge par rapport aux alluvions fines. On a, d'autre part, affecté à ces dernières les deux ensembles suivants de caractéristiques mécaniques :

$$
\begin{array}{lll}
\gamma=17,5 \mathrm{kN} / \mathrm{m}^{3}, & c^{\prime}=0, & \varphi^{\prime}=35^{\circ} \\
\gamma=14 \mathrm{kN} / \mathrm{m}^{3}, & c^{\prime}=0, & \varphi^{\prime}=40^{\circ}
\end{array}
$$

Les conditions hydrauliques du calcul correspondaient aux trois positions $N_{1}, N_{2}, N_{3}$ de la surface de la nappe amont, le niveau de la rivière étant celui constaté lors de la rupture.

Les poussée et butée à l'équilibre limite ont été calculées par la méthode de Coulomb, la configuration relativement complexe du site n'ayant pas permis d'utiliser les tables et abaques usuels. Les valeurs adoptées pour l'inclinaison $\delta$ des contraintes sur I'horizontale étaient respectivement $\delta_{\mathrm{p}}=0$ dans le cas de la poussée ${ }^{(*)}$ et $\delta_{p}=-\frac{2}{3} \varphi^{\prime}$ dans le cas de la butée.

(") La poussée totale $\mathrm{Pa}$ a été déterminée au moyen d'un logiciel permettant de calculer la poussée sur un écran lisse vertical. Ce logiciel est applicable à des stratifications complexes comportant au maximum 5 couches de caractéristiques différentes. La surface du sol ainsi que les surfaces de séparation des couches peuvent être quelconques. Le sol peut être baigné par une nappe statique ou en mouvement (dans ce dernier cas, les pressions interstielles sont calculées en supposant les équipotentielles verticales). La distribution des contraintes sur l'écran est obtenue par dérivation de la poussée totale exprimée en fonction de la hauteur de l'écran. 
La résistance théorique à l'arrachement de chaque tirant, déterminée à partir des résultats des essais pressiométriques effectués lors de l'étude initiale du projet, était d'environ $300 \mathrm{kN}$. Les tirants étant espacés de $4.5 \mathrm{~m}$ et inclinés de $30^{\circ}$ sur l'horizontale, la valeur approximative de $T_{\max }$ était :

$$
T_{\max }=\frac{300 \times \cos 30^{\circ}}{4,5}=58 \mathrm{kN} \text {. }
$$

Les valeurs des coefficients de sécurité $\mathrm{Fa}$ et $\mathrm{Fp}$ correspondant aux hypothèses successivement envisagées sont récapitulées dans le tableau 3.

On constate que toutes les valeurs de $\mathrm{Fa}$ sont supérieures à l'unité, la seule vraiment critique $(1,07<\mathrm{Fa}<1,13)$ n'étant obtenue qu'en combinant les hypothèses les plus pessimistes (remblai sans cohésion et remontée maximale de la nappe arrière). En revanche, la seule valeur de $\mathrm{Fp}$ compatible avec la stabilité de l'ouvrage $(1,44<F p<1,55)$ n'est obtenue qu'en admettant que le remblai se comporte comme un matériau très cohérent et que la fuite à la canalisation ne provoque pas de remontée de la nappe arrière. On notera d'ailleurs que, dans ce cas, qui correspond à la prise en compte simultanée des hypothèses les plus favorables, la valeur de Fp est largement inférieure à la valeur 2 généralement adoptée pour la détermination de la fiche d'un rideau de palplanches.

\begin{tabular}{|c|c|c|c|c|c|}
\hline \multirow{3}{*}{$\begin{array}{l}\text { Alluvions } \\
\text { fines }\end{array}$} & \multirow{3}{*}{$\begin{array}{l}\text { Nappe } \\
\text { interne }\end{array}$} & \multicolumn{4}{|c|}{ Remblai } \\
\hline & & \multicolumn{2}{|c|}{$c^{\prime}=0, \varphi^{\prime}=36^{\circ}$} & \multicolumn{2}{|c|}{$c^{\prime}$ grand } \\
\hline & & $F p$ & $\mathrm{Fa}$ & Fp & $\mathrm{Fa}$ \\
\hline$\gamma=17,5 \mathrm{kN} / \mathrm{m}^{3}$ & $N$, & 0,86 & 1,76 & 1,44 & 4,12 \\
\hline$c^{\prime}=0$ & $\mathrm{~N}_{2}$ & 0.72 & 1,48 & 1,15 & 3,72 \\
\hline$\varphi^{\prime}=35^{\circ}$ & $\mathrm{N}_{3}$ & 0,64 & 1,07 & 0,89 & 2,36 \\
\hline$\gamma=14 \mathrm{kN} / \mathrm{m}^{3}$ & $N_{1}$ & 0,89 & 1,90 & 1,55 & 4.18 \\
\hline$c^{\prime}=0$ & $\mathrm{~N}_{2}$ & 0.73 & 1.61 & 1.21 & 3.92 \\
\hline$\phi^{\prime}=40^{\circ}$ & $\mathrm{N}_{3}$ & 0,62 & 1,13 & 0,87 & 2,47 \\
\hline
\end{tabular}

Tableau 3 Etude de la stabilité propre du rideau de palplanches

II s'ensuivait donc que la fiche du rideau était insuffisante et que, même dans les conditions normales de service, le sol au contact de la face avant était dans un état voisin de l'équilibre limite (ceci était confirmé par la fissuration de la chaussée constatée en amont de la zone effondrée). L'ouvrage ayant cependant été stable pendant 18 mois la rupture intervenue avait vraisemblablement été déclanchée par une remontée locale de la nappe arrière liée à la fuite de la canalisation de distribution d'eau.

\section{Réparation et confortement de l'ouvrage}

La zone de rupture se situait approximativement au milieu d'une section de $500 \mathrm{~m}$ de longueur, dans laquelle les caractéristiques du rideau de palplanches et celles du remblai (hauteur et pente du talus) ne subissaient pas de variations sensibles. L'étude précédente avait montré que, dans la partie amont de cette section où l'on avait constaté une fissuration de la chaussée et dans la zone effondrée, le rideau de palplanches était sérieusement sous-dimensionné. Se posait alors la question des conditions de stabilité de la partie aval de la section dans laquelle le rideau, le remblai et la chaussée ne présentaient aucun signe d'instabilité. Pour y répondre, une étude géotechnique complémentaire basée sur 6 sondages, a été entreprise. Elle a fait apparaître que, dans cette zone, la coupe et les caractéristiques du sol étaient semblables à celles obtenues plus en amont, c'est-à-dire que l'équilibre du rideau y était également précaire. II était donc nécessaire, non seulement de réparer la zone effondrée, mais aussi de conforter le rideau encore stable sur une longueur d'environ $200 \mathrm{~m}$ de part et d'autre de cette dernière.

La solution reteniue pour la remise en état de la zone effondrée a consisté à substituer au rideau détruit un rideau de palplanches de caractéristiques adaptées, fiché dans le sable de Beauchamp et ancré en tête par des tirants précontraints inclinés à $45^{\circ}$, scellés dans les marnes et caillasses. Pour conforter le rideau encore intact, on a choisi de le doubler par un rideau de palplanches de même caractéristiques que celui mis en place dans la zone de rupture, étayé par un système d'ancrage identique. Il était prévu de battre le rideau confortatif à environ $1 \mathrm{~m}$ en avant du rideau initial et de combler l'intervalle entre les deux rideaux à leur partie supérieure au moyen d'un blocage en béton coulé avant mise en place des tirants (fig. 8), ceux-ci devant être forés à travers ce blocage et à travers le rideau initial.

Le rideau confortatif a été calculé par la méthode de Rowe (1952, cité par Josseaume, 1974) applicable à un matériau graveleux, dans laquelle:

- la fiche du rideau et la réaction d'ancrage sont déterminées par un calcul en butée simple prenant en compte sur la face avant, les $\frac{2}{3}$ de la butée de Rankine;

- la valeur du moment fléchissant maximum est la valeur donnée par le calcul en butée simple. affectée d'un coefficient minorateur qui est fonction de la flexibilité du rideau et de l'état de compacité du sol.

Les calculs ont été effectués en tenant compte de la coupe du sol au niveau du profil de sondages 3 (le plus défavorable) sur la base des caractéristiques géotechniques relativement pessimistes données dans le tableau 4 . On, en outre, considéré que la nappe arrière affleurait la tête du rideau et que la rivière atteignait son niveau le plus bas, qui s'établissait à $1,50 \mathrm{~m}$ au-dessus de cette dernière. On signalera par ailleurs :

- que la poussée sur la face arrière a été calculée par la méthode de Coulomb dans l'hypothèse $\delta \mathrm{a}=0$;

- que, compte tenu de la faible déformabilité du sable de Beauchamp vis-à-vis des alluvions qui le surmontent, celles-ci ont été considérées comme une simple surcharge dans le calcul de la butée sur la face avant. 


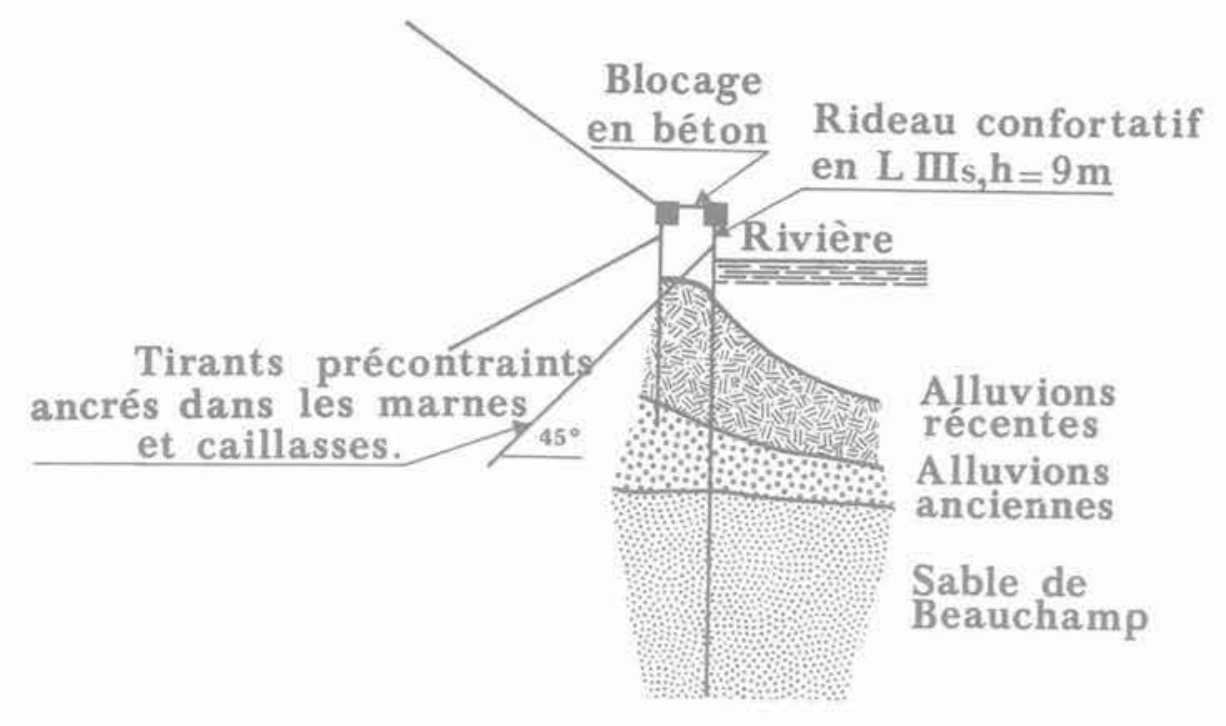

Fig. 8 Principe de fonctionnement du rideau de part et d'autre de la zone de rupture.

\begin{tabular}{l|c|c|c}
\hline Formation & $\begin{array}{c}\gamma \\
\left(\mathrm{kN} / \mathrm{m}^{3}\right)\end{array}$ & $\begin{array}{c}c^{\prime} \\
(\mathrm{kPa})\end{array}$ & $\begin{array}{c}\varphi^{\prime} \\
\left({ }^{\circ}\right)\end{array}$ \\
\hline Remblai & 20 & 0 & 35 \\
\hline Alluvions & 17,5 & 0 & 30 \\
\hline Sable de Beauchamp & 20 & 0 & 39 \\
\hline
\end{tabular}

Tableau 4 Caractéristiques géotechniques prises en compte dans le calcul du rideau confortatif

Les résultats du calcul ont conduit à réaliser le rideau confortatif en paiplanches Larssen IIIs de longueur $9 \mathrm{~m}$ (telle qu'après battage, le pied du rideau se situe au niveau du toit des marnes et caillasses). Le calcul indiquait également que, compte tenu de l'espacement de $3 \mathrm{~m}$ choisi pour les tirants, chacun d'eux devrait équilibrer une tension de service de $650 \mathrm{kN}$. Les tirants mis en œuvre ont été des tirants de type TMD (procédé SIF-Entreprise Bachy). Deux séries de 3 essais de tirants effectués avant la construction de l'ouvrage ont conduit à adopter une longueur de scellement de $9 \mathrm{~m}$ dans les marnes et caillasses qui constituaient la couche d'ancrage.

Un problème relatif au mode d'exécution des travaux s'est posé dans la zone effondrée où les alluvions fines avaient été fortement remaniées lors de la rupture. Elles étaient, de ce fait, trop compressibles pour que le rideau ne subisse pas de déformations excessives lors de la précontrainte des tirants et pour que le remblai routier reconstruit après cette opération ne tasse de façon inacceptable. On ne pouvait substituer un sol de bonne qualité aux alluvions remaniées, car leur curage sur une épaisseur de 3 à $4 \mathrm{~m}$ était susceptible, entre autres conséquences, de provoquer de nouveaux glissements dans la zone endommagée. Dans ces conditions, seule l'amélioration du sol en place pouvait être envisagée. La solution choisie a été la consolidation des alluvions par pieux-colonnes ballastés, technique déjà employée avec succès dans un site analogue (Jardin, 1974). Les pieux, profonds de $5 \mathrm{~m}$, ont été implantés suivant un maillage de $2 \mathrm{~m} \times 2 \mathrm{~m}$ sauf à proximité du rideau où un maillage plus lâche $(3 \mathrm{~m} \times 3 \mathrm{~m})$ a été adopté afin de ne pas gêner l'exécution ultérieure des tirants. Cette méthode a donné entière satisfaction.

\section{Références bibliographiques}

Faure R. M., Moreau M., Gauriat M., Gueniot M., (1975) "Application de la visualisation interactive aux calculs de stabilité des pentes. Programme Vestal $n$. Bull. Liaison des Labo. des P. et Ch. $n^{\circ} 78$, Juillet-Août 1975 , pp. 117-130.

Jardin J., (1974) «Traitement d'alluvions compressibles par pieux-colonnes ballastés . Bull. Liaison des Labo. des $P$, et Ch. $n^{\circ} 69$, Janvier-Février 1974 , p. 30.

Josseaume H., (1974) « Méthodes de calcul des rideaux de palplanches. Étude bibliographiquen. Bull. Liaison des Labo. des P. et Ch. $n^{\circ} 72$, Juillet-Août 1972 , pp. 177-212.

Rowe P.W., (1952) «Anchored sheet-pile walls". Institution of Civil Engineers, Proceedings, London, vol. 1 , january 1952 , pp. 27-70. 
